\title{
Searching for the Signatures of Terrestrial Planets in "Hot" Analogs
}

\author{
J. I. González Hernández ${ }^{1,2}$, E. Delgado Mena ${ }^{1,2}$, G. Israelian ${ }^{1,2}$, S. G. \\ Sousa $^{3,4}$, N. C. Santos ${ }^{3,4}$, and S. Udry ${ }^{5}$ \\ ${ }^{1}$ Instituto de Astrofísica de Canarias, E-38200 La Laguna, Tenerife, Spain. \\ email: jonay@iac.es \\ ${ }^{2}$ Departamento de Astrofísica, Universidad de La Laguna, 38205 La Laguna, Tenerife, Spain. \\ ${ }^{3}$ Centro de Astrofísica, Universidade do Porto, Rua das Estrelas, 4150-762 Porto, Portugal. \\ ${ }^{4}$ Departamento de Física e Astronomia, Faculdade de Ciências, Universidade do Porto, \\ Portugal. \\ ${ }^{5}$ Centro de Física Computacional, Universidade de Coimbra, Portugal. \\ ${ }^{6}$ Observatoire Astronomique de l'Université de Genève, 51 Ch. des Maillettes, Sauverny, \\ Ch1290 Versoix, Switzerland
}

\begin{abstract}
The Sun has been suggested to have a slightly low refractory-to-volatile abundance ratio when compared with field solar twins. This result may be interpreted as due to the fact that the refractory elements were trapped in rocky planets at the formation of the Solar System.

A detailed and differential chemical abundance study was already performed in order to investigate this hypothesis in solar analogs with and without detected planets using high-resolution and high-S/N HARPS and UVES spectra of a relatively large sample of solar analogs with and without planets. We obtained very similar behaviours for both samples of stars with and without planets, even for two stars with super-Earth-like planets, which may indicate that this solar trend may not be related to the presence of terrestrial planets.

The depletion signature should be imprinted once the convection zone reaches the current size. This suggests that stars hotter than the Sun should show this effect enhanced, due to their narrower convective zone. However, to avoid non-LTE, 3D, and other effects, we need to identify "hot" analogs with a $T_{\text {eff }} \sim 6100 \mathrm{~K}$, to perform a differential analysis.

Here, we present the preliminary results of our analysis using HARPS and UVES highresolution and high-S/N spectra of a sample of $\sim 60$ "hot" analogs with and without planets, trying to search for some "hot" reference analogs.
\end{abstract}

Keywords. stars: abundances — stars: atmospheres — stars: fundamental parameters — stars: planetary systems - stars: planetary systems: formation

\section{Introduction: $[\mathrm{X} / \mathrm{Fe}]$ vs. $T_{C}$}

In the last few years, the chemical abundances of heavy elements in solar analogs have been extensively investigated. Meléndez et al. (2009) found in a sample of 11 solar twins a well-defined trend $[\mathrm{X} / \mathrm{Fe}]$ with respect to the condensation temperature, $T_{C}$, which was interpreted as the result of the formation of terrestrial planets in the solar system (see also Ramírez et al. 2009, 2010).

A detailed chemical analysis of very high-quality HARPS and UVES spectroscopic data did not show any significant difference between the abundance ratios of a sample of 95 solar analogs with and without planets (see González Hernández et al. 2010). In addition, two stars of the HARPS sample hosting Super-Earth like planets do not seem to provide clear evidence of terrestrial planets in their abundance patterns relative to iron (see González Hernández et al. 2011). In particular, after correcting for the Galactic 
chemical evolution effects, both stars do not seem to show any trend. This may indicate that there is no signature of terrestrial planets in these two stars even if there has been already detected one Super-Earth-like planet orbiting each of these stars.

\section{Identifying the "hot" reference analog}

According to Meléndez et al. (2009), the formation of terrestrial planets in a stellar planetary system would cause a depletion of refractory (with high $T_{C}$ values) elements with respect to volatiles (with low $T_{C}$ values) in the convective envelope of the star. However, the convective zone would have to reach the present state early enough (i.e. after $\sim 20$ Myr for a solar-type star) to be able to show the depletion signature.

This possible signature would, in principle, be stronger in stars hotter than the Sun, due to their shallower convective zones. Thus, we have inspected the HARPS GTO sample of stars in the temperature range $5950-6350 \mathrm{~K}$ and found about 60 stars with and without planets with very high-quality UVES and HARPS spectroscopic data.
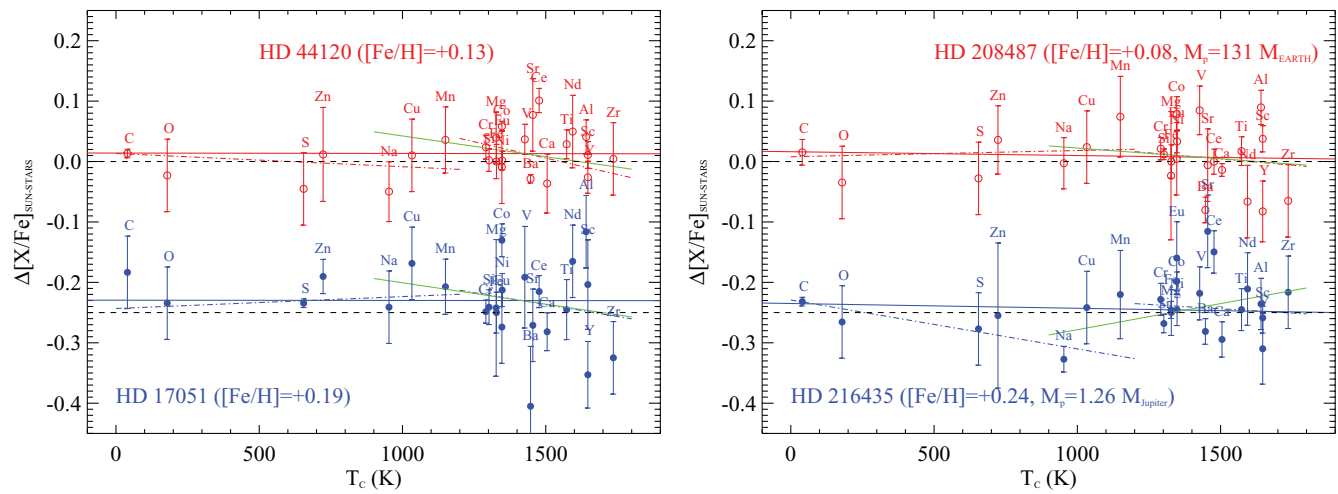

Figure 1. Left panel: Abundance differences, $\Delta[\mathrm{X} / \mathrm{Fe}]_{\mathrm{SUN}-\mathrm{STARS}}$, between the Sun, and 2 "hot" analogs without planets from the HARPS GTO sample. Each element abundance ratio has been corrected using a linear fit to the Galactic chemical trend of the corresponding element at the metallicity of each star. Linear fits for different $T_{C}$ ranges to the data points weighted with the error bars are also displayed. We note the different slopes derived when choosing the range $T_{C}>1200 \mathrm{~K}$ (dashed-dotted line) as in Meléndez et al. (2009) and González Hernández et al. (2010), and $T_{C}>900 \mathrm{~K}$ (dashed-three-dotted line) as in Ramírez et al. (2009, 2010). Right panel: Same as left panel of this figure but for two "hot" planet-host stars. An arbitrary shift of -0.25 dex has been applied, in both panels, to the abundances of the element abundances in the stars HD 17051 and HD 216435.

In the left panel of Fig. 1, we display the abundances of two "hot" stars without planets at $T_{\text {eff }} \sim 6100 \mathrm{~K}$ and some linear fits for different $T_{C}$ ranges. We have already removed the Galactic chemical evolution effects with the aim to find stars with similar abundance trends than the Sun to use them as references. In the right panel of Fig. 1, we display two possible "hot" reference stars hosting planets.

\section{References}

González Hernández, J. I., Israelian, G., Santos, N. C., et al., 2010, ApJ, 720, 1592

González Hernández, J. I., et al., 2011, IAU Symposium 276 (arXiv:1011.6125)

Meléndez, J., Asplund, M., Gustafsson, B., \& Yong, D. 2009, ApJ Letters, 704, L66

Ramírez, I., Meléndez, J., \& Asplund, M. 2009, A\&\&A Letters, 508, L17

Ramírez, I., Asplund, M., Baumann, P., Meléndez, J., \& Bensby, T. 2010, A\&3A, 521, A33 